\title{
Overview of Swarm Robotics
}

\author{
Ms. Heena S. Pabani \\ Prof. Atul N. Shire \\ Mb:8605159427 email: heena.pabani@gmail.com \\ Department of Electronics and Telecommunication \\ Jawaharlal Darda Institute of Engineering and Technology \\ Yavatmal, India
}

\begin{abstract}
The swarm robotics inspired from nature is a combination of swarm intelligence and robotics, which shows a great potential in several aspects. It is important to study swarm robotics system because it has desirable properties unlikely to be found in other systems, for example they scale very well; there is no single point of failure, making swarm systems very robust and well suited in operating in safety critical situations. As research progresses in robot system, more and more aspects are explored in multi robot system. This paper describes advances in multi robot system and discusses the current state of art. The focus is principally on the research that has been demonstrated in various entity projects.
\end{abstract}

\section{INTRODUCTION}

The field of swarm robotics has its origin in the late 1980's [1]when researchers began investigating issues in multi robot systems. Swarms have many advantages such as enhanced task performance, high reliability (fault tolerance), low unit complexity and less cost over traditional robotic systems. It is possible to accomplish some tasks that would be impossible for a single robot to achieve. Swarm-bots are a collection of mobile robots able to self-assemble and to self-organize in order to solve problems that cannot be solved by a single robot[2]. These robots bring together the power of swarm intelligence with the flexibility of self-reconfiguration as aggregate swarm-bots can dynamically change their structure to match environmental variations. Swarm robots are more than just networks of independent agents; they are potentially reconfigurable networks of communicating agents capable of coordinated sensing and interaction with the environment.

Biological inspiration

Since the swarm robotics is mostly inspired from the nature swarms, it's a good reference for analyzing the characteristics of nature swarms. The research of swarm robotics started a century ago. The first hypothesis is quite personified [10] and assumes that each individual has a unique ID for cooperation and communication. The information exchange in the swarm is regarded as a centralized network. The queens in ant and bee colonies are supposed to be responsible for transmitting and

assigning the information to each agent [11]. However, Jha, et al.[12] proved that the network in the swarm is decentralized. Thanks to the research in recent half century, the biologists can now assert that there are no unique IDs or other

globally storage information in the network. No single agent can access to all the information in the network and a pacemaker is therefore inexistent.

\section{What is Swarm intelligence?}

As an emerging research area, the swarm intelligence has attracted many researchers' attention since the concept was proposed in 1980s. It has now become an interdisciplinary frontier and focus of many disciplines including artificial intelligence, economics, sociology, biology, etc. It has been observed a long time ago that some species survive in the cruel nature taking the advantage of the power of swarms, rather than the wisdom of individuals. The individuals in such swarm are not highly intelligent, yet they complete the complex tasks through cooperation and division of labor and show high intelligence as a whole swarm which is highly selforganized and self-adaptive. The individuals can be regarded as agents with simple and single abilities. Some of them have the ability to evolve themselves when dealing with certain problems to make better compatibility.

\section{LITERATURE REVIEW}

This section details and explains research that has been previously carried out in the subject area of swarm robotics including four entity projects:

(i)Kobot Robotic System

(ii) Kilobot Project

(iii)e-puck project 
(iv)irobot project

\section{(i)THE KOBOT ROBOTIC SYSTEM}

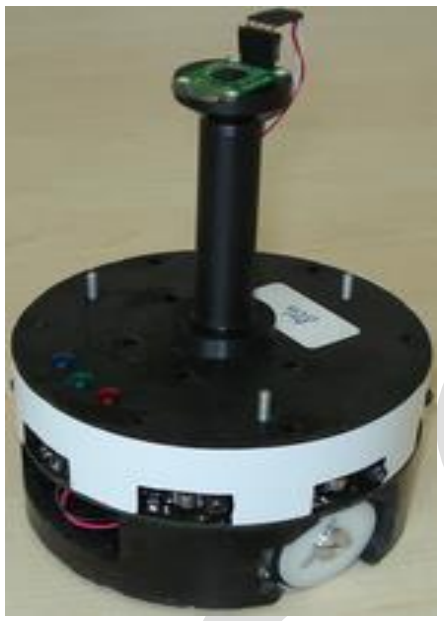

Fig 1: A picture of the KOBOT

Ali E. Turgut et al in their paper mentions two challenges for the Swarm Robotics system to be used in real world application [13]. First is the need for large numbers of robots, which settles for no less than the means of well-established mass production. Second is the need for robust, flexible and scalable coordination methods to operate on swarm robotic systems.

The requirements of a mobile robot to be used as part of a swarm robotic system differ from that of a mobile robot to be used as standalone. The challenges require a platform for the researchers, that would facilitate study, rather than interfering with it, and allow the researchers to concentrate on the problems of coordination.

The parameters to be considered for mobile robot over standalone are:

- Sensing and Signalling

- Interference from environmental factors:

- Kin-detection

- $\quad$ Stigmergic sensing and signalling

- Communication

- Physical interaction

- Power

- Size

- Cost

A new robot platform "KOBOT" is designed to tackle most of these requirements.

Kobot mobile robot platform is specifically designed for swarm robotic studies. The robot has the size of a CD (diameter of $12 \mathrm{~cm}$ ), a weight of 350 grams, and it is differentially driven by two high quality DC motors. It has IR sensors for kin and obstacle detection and a digital compass for heading measurement. An IEEE 802.15.4/ZigBee compliant XBee wireless module with a range of approximately $20 \mathrm{~m}$ indoors can be used for communication between robots and between the robots and a computer console. The robot hosts a $20 \mathrm{MHz}$ PIC18F4620A microcontroller. Batteries are designed to be light, small, but extendable and power-efficient and relatively cheap robot platform for swarm robotics research.

Kobot's body consists of : (1) a cylindrical base, which houses the motors, the battery pack and the short-range sensor board, and (2) a cylindrical cap that covers the body. Both pieces are manufactured by casting polyurethanes, a low-density material that is ideal to create a light yet durable body structure for the robot. The cap is wrapped with white paper, to increase the "visibility" of the robots to each other. High efficiency (to save power), low profile (to save space), high torque DC gear-head motors are used which are directly connected to the wheels. The motors are driven using high switching frequency Si9988 motor drivers. 
International Journal of Engineering Research and General Science Volume 3, Issue 1, January-February, 2015 ISSN 2091-2730

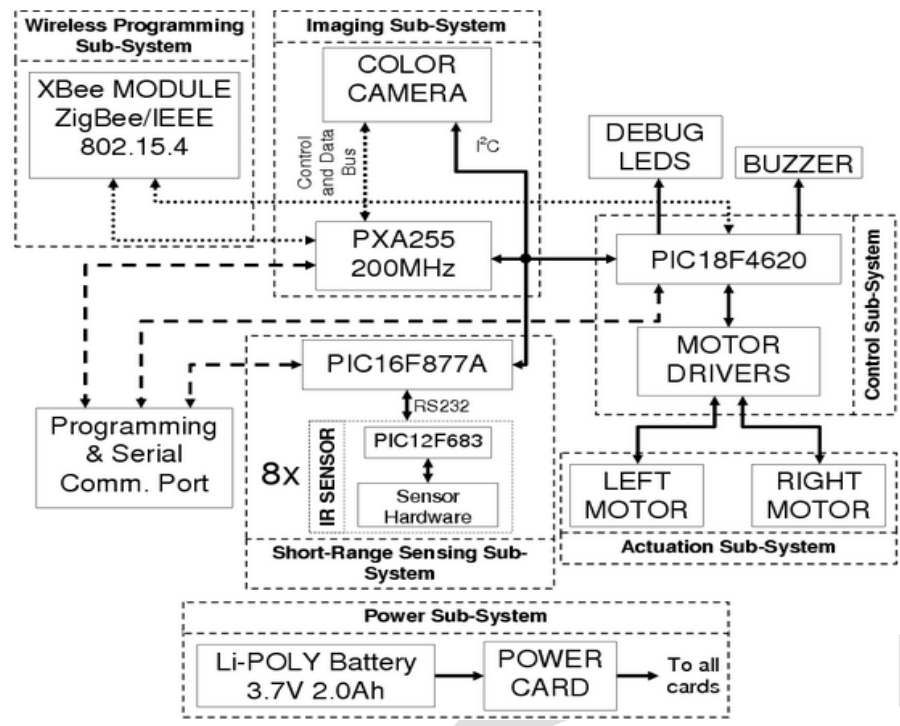

Fig 2:Block diagram for kobot

The overall system design of Kobot is shown in Figure 2. At the heart of the Kobot, there is the control sub-system to which all of the information from the other sub-systems, that is short range sensing, communication, vision and power, are fed to the control subsystem. With the information acquired, a 20MHz PIC16F877A, called the main controller, implements the control algorithm which determines the behaviors of Kobot.

There are several items that can be regarded as future work. Imaging sub-system is yet to be fully integrated, as an optional sensing system. A new short-range sensing system is planned to be designed which will be faster, more modular and will enable short-range communication between nearby Kobot's. Communication among Kobot's will also be enabled using ZigBee protocol.

\section{(ii)KILOBOT}

The Kilobot is designed to make tests of collective algorithms on hundreds or thousands of robots accessible to robotics researchers. Kilobot is a simple modular robot that is designed to work in a collective to self-assemble and self-heal that collective's shape. Though the Kilobots are low-cost, they maintain abilities similar to other collective robots. These abilities include differential drive locomotion, on-board computation power, neighbor-to-neighbor communication, neighbor-to-neighbor distance sensing, and ambient light sensing. Additionally they are designed to operate such that no robot requires any individual attention by a human operator. This makes controlling a group of Kilobots easy, whether there are 10 or 1000 in the group.

Michael Rubenstein et al in their paper have given the algorithm that allows a simulated collective of robots to self-assemble and selfheal a desired shape, keeping the shape sized proportional to the number of robots in the collective[14].

In previous work [3,4] a distributed control method called S-DASH was presented which enables a collective of robots to form a given shape at a scale proportional to the total number of robots. If the shape of the collective is damaged, for example by removing some robots, then S-DASH will reform the shape at a new, smaller scale, proportional to the new number of robots.

In the previous work of S-DASH, the collective behaviors are demonstrated only in simulation. These simulated robots are very simple in their capabilities. They are capable of moving forward, rotating, communicating with local neighbors, and measuring the distance between themselves and their local neighbors. Many robot platforms exist that have these capabilities, for example [5,6,7], and therefore in theory are capable of demonstrating S-DASH. However, these robots are not practical to operate as a collective on the order of $2^{10}$ robots.

For an example of why they are not practical in such large numbers, consider the simple task of powering on the robots in the collective. With a standard robot, for example the E-PUCK [7] turning on the robot requires a user to toggle a power switch located on the side of the individual robot. If one robot could be powered up this way on average every 2 seconds, it would still take a single user over 30 minutes to power on all 1024 robots in the collective! Some other reasons these robots are not practical for such a large scale collective include: robot cost, operability (powering, charging, programming, etc...), and physical size.

The Kilobot module is designed to meet the requirements of S-DASH, while at the same time easily operate in large collectives (more than 210 robots). The requirements of S-DASH are that the robot be able to move forward, turn, communicate with neighbors, and measure distances to neighbors. Kilobot meets these requirements, while keeping the design balanced against other needs for operating a large collective, such as keeping the cost per robot under \$15(US), and ease in programming. Fig. 3 shows a prototype version of Kilobot.

The Kilobot is designed to provide scientists with a physical testbed for advancing the understanding of collective behavior and realizing its potential to deliver solutions for a wide range of challenges. 
The Kilobot module is designed to meet the requirements of S-DASH, while at the same time easily operate in large collectives (more than 210 robots). The requirements of S-DASH are that the robot be able to move forward, turn, communicate with neighbors, and measure distances to neighbors. Kilobot meets these requirements, while keeping the design balanced against other needs for operating a large collective, such as keeping the cost per robot under \$15(US), and ease in programming. Fig. 3 shows a prototype version of Kilobot.
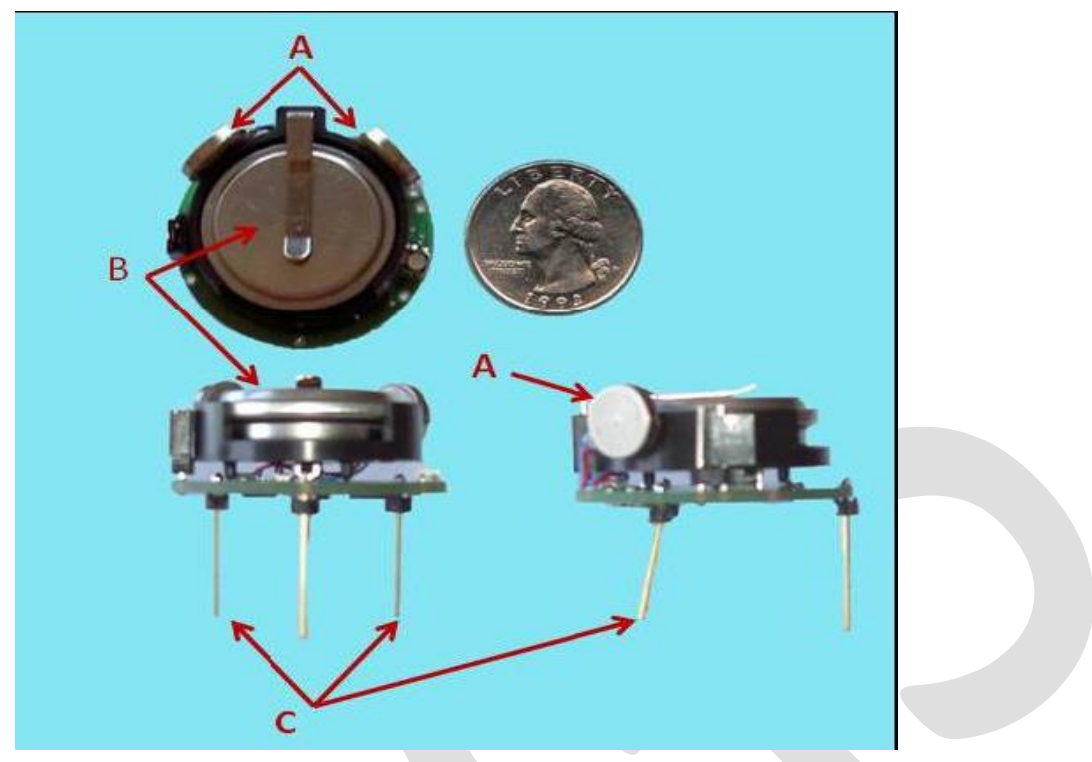

Figure 3. A prototype Kilobot module (top view in upper left, front view in lower left, side view in lower right) next to a US quarter for scale. Some features are (A) vibration motors, (B) battery, (C) slanted legs.

The Kilobot swarm is a thousand-robot swarm designed to allow one to program and experiment with collective behaviors in largescale autonomous swarms. Each robot has the basic capabilities required for an autonomous swarm robot (programmable controller, basic locomotion, and local communication), but is made with low-cost parts and is mostly assembled by an automated process. In addition, the system design allows a single user to easily and scalably operate a large Kilobot collective, such as "hands-off" programming, powering on, and charging all robots.

Charging:

Each robot has a built in lithium ion battery charger, that will charge the on board battery when 6 volts is applied to the two slanted legs. The charger will automatically cease charging when the battery becomes full. This allows bulk charging of the Kilobots by placing each robot on a set of conducting strips attached to a $6 \mathrm{v}$ power supply, as visualized in Fig 4 . This charging method is inspired from [8].

(iii) e-puck:

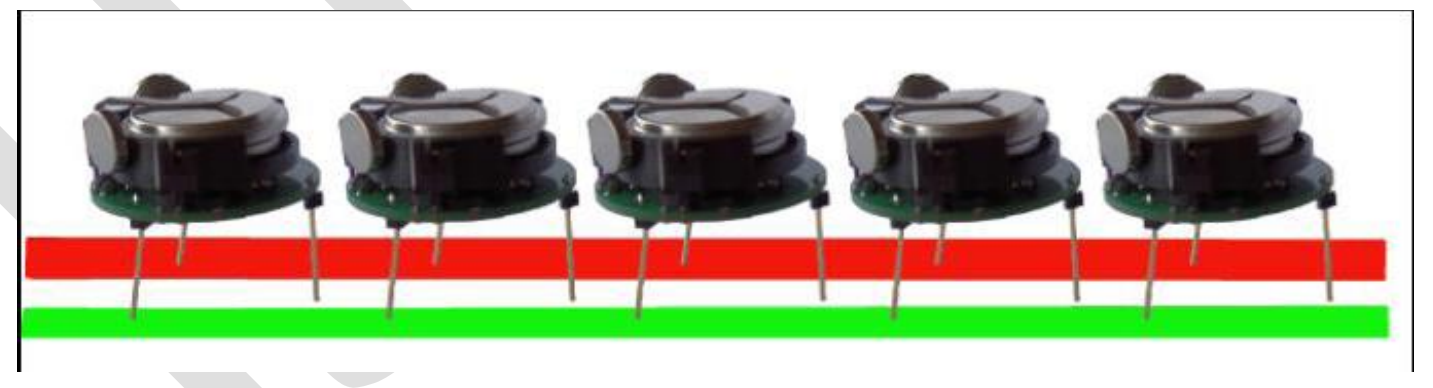

Figure 4. A visualization of the Kilobot charging scheme.

e-puck is the latest mini mobile robot developed at the Swiss Federal Institute of Technology in Lausanne (EPFL) for teaching purposes. It is now commercially available from GCtronic.

e-puck is powered by a dsPIC processor and features a very large number of sensors in its basic configuration. The e-puck hardware and software is fully open source (www.e-puck.org) providing low-level access to every electronic device and offering unlimited extension possibilities.

- Microcontroller - dsPIC at $60 \mathrm{MHz}$ (15MIPS), DSP core for signal processing

- Sensors - 8 proximity and ambient light, 3D accelerometer, 3 omni-directional microphones, VGA color camera, IR receiver for remote control

- Outputs - 8 red leds, green body light, 1 strong red LED in front, speaker (WAV files) 
International Journal of Engineering Research and General Science Volume 3, Issue 1, January-February, 2015 ISSN 2091-2730

- Communication - RS232 and Bluetooth connection and programming

- C programming - free C compiler, GNU GCC, Webots simulator

- Battery - 5 WH Lilon battery for $\sim 3$ hours autonomy

- Low price - 850 CHF ( $\sim 570 €, \sim 850 \$)$. Quantity discount available.

Mobile robots have the potential to become the ideal tool to teach a broad range of engineering disciplines. Indeed, mobile robots are getting increasingly complex and accessible. They embed elements from diverse fields such as mechanics, digital electronics, automatic control, signal processing, embedded programming, and energy management. Moreover, they are attractive for students which increase their motivation to learn. However, the requirements of an effective education tool bring new constraints to robotics. Thanks to its particular design, the e-puck can be used in a large spectrum of teaching activities, not strictly related to robotics. The epuck fits this purpose and is appreciated by 90 percent of a large sample of students.

Francesco Mondada et al have for the first time, presented the design approach resulting in the e-puck, an educational desktop mobile robot developed at the 'Ecole Polytechnique F'ed'erale de Lausanne (EPFL) for a broad exploitation in teaching activities[15].

At EPFL,[9] several courses exploit the e-puck robot as experimentation platform. Practical excesises are organized in following teaching areas:

- Signal processing.

- Automatic control.

- Behaviour-based robotics.

- Distributed intelligent systems.

- Position estimation and path finding of a mobile robot.

Table 1: Ongoing courses

\begin{tabular}{|l|l|l|}
\hline Courses using e-puck & Laboratory & school \\
\hline Biological and artificial intelligent system & Laboratory of intelligent system & EPFL \\
\hline Robots mobile & Laboratory of intelligent system & EPFL \\
\hline Swarm intelligence & Swarm-intelligent group & EPFL \\
\hline
\end{tabular}

More than 200 students over 3 years have validated this concept and shown their satisfaction using the e-puck. The open-source nature of this robot improves the quality of the support to the students by providing full access to knowledge at every level. For teachers this simplifies maintenance and opens new experimentation possibilities. Finally, the rapid diffusion of the e-puck in the research community shows its versatility as a scientific experimentation tool.

\section{(iv)IROBOT PROJECT}

The iRobot Swarm project represents the state of the art in algorithms, hardware, and user interfaces for large swarms of autonomous robots. The goal of the project is to develop distributed algorithms for robotic swarms composed of hundreds of individual robots. Programs for individual robots need to be robust to complex real-world environments, and the group software needs to be tolerant to the addition or failure of any number of individuals.

For co-operating for cooperating over 100 robots iRobot Swarm Project [94] is projected by MIT. James McLurkin et al in their paper have described a set of distributed algorithms that can be used to disperse a large group of autonomous mobile robots efficiently throughout an indoor environment[16].

Also in dangerous situations like earthquake and fire it is not always possible for a rescue team to enter a building immediately, due to safety concerns for the human rescuers. However, a team of small robots could be deployed to explore the building, locate survivors, and mark pathways to the exits. This information can then be relayed back to the human search and rescue team. Elizabeth Jensen et al in her paper has proposed a rolling dispersion algorithm, which makes use of a small number of robots and achieves full exploration[17]. 
International Journal of Engineering Research and General Science Volume 3, Issue 1, January-February, 2015 ISSN 2091-2730

\section{CONCLUSION}

In this paper we have first discussed the biological inspiration for swarm robotics and the role of swarm intelligence. Further, few of the entity projects have been discussed-kobot, kilobot, e-puck and irobot. These are a few of the projects undertaken by various researchers to explore the possibilities of introducing swarm robots in real life.

\section{FUTURE SCOPE}

Robot swarms might one day tunnel through rubble to find survivors, monitor the environment and remove contaminants, assist dwindling bee populations in pollinating crops, and self-assemble to form support structures in collapsed buildings. Swarms of robots acting together to carry out jobs could provide new opportunities for humans to harness the power of machines.They could play a part in military, or search and rescue operations, acting together in areas where it would be too dangerous or impractical for humans to go. In industry too, robot swarms could be put to use, improving manufacturing processes and workplace safety.

Researchers are developing Artificial Intelligence to control robots in a variety of ways. The key is to work out what is the minimum amount of information needed by the robot to accomplish its task. That's important because it means the robot may not need any memory, and possibly not even a processing unit, so this technology could work for nano scale robots, for example in medical applications.

\section{REFERENCES:}

[1] Lynne E. Parker in "Current Research in multi robotic system."

[2] Ying Tang, Zhong Yang ZHENG in "Research Advance in swarm robotics"

[3] Rubenstein, M., Shen, W-M. "Scalable Self-Assembly and Self-Repair In A Collective Of Robots" IROS, Oct 2009.

[4] Rubenstein, M., Shen, W-M. "Automatic Scalable Size Selection for the Shape of a Distributed Robotic Collective" IROS, Oct 2010.(submitted)

[5] Chiu, H., Rubenstein, M., Shen, W-M. "Deformable Wheel'-A Self-Recovering Modular Rolling Track." Intl. Symposium on Distributed Robotic Systems. Tsukuba, Japan, 2008.

[6] P. Maxim, S. Hettiarachchi, W. Spears, D. Spears, J. Hamman, T. Kunkel, C. Speiser. Trilateration localization for multi-robot teams. Sixth International Conference on Informatics in Control, Automation and Robotics, Special Session on Multi-Agent Robotic Systems. 2008.

[7] Mondada, F., Bonani, M., Raemy, X., Pugh, J., Cianci, C., Klaptocz, A., Magnenat, S., Zufferey, J.-C., Floreano, D. and Martinoli, A. (2009) The e-puck, a Robot Designed for Education in Engineering. Proceedings of the 9th Conference on Autonomous Robot Systems and Competitions, 1(1) pp. 59-65.

[8]S. Kornienko, O. Kornienko, A. Nagarathinam, and P. Levi. From real robot swarm to evolutionary multi-robot organism. In Proc. of the CEC2007, Singapore, 2007.

[9] The e-puck, a Robot Designed for Education in Engineering

Francesco Mondada1, Michael Bonani1, Xavier Raemy2, James Pugh2, Christopher Cianci2,

Adam Klaptocz3, St'ephane Magnenat1, Jean-Christophe Zufferey3, Dario Floreano3, Alcherio Martinoli2

[10] Büchner L. La vie psychique des be^tes. Paris: C Reinwald; 1881.

[11] Reeve HK, Gamboa GJ. Queen regulation of worker foraging in paper

wasps: a social feedback control system (polistesfuscatus, hymenoptera:

Vespidae). Behaviour 1987;102(3/4):147e67.

[12] Jha S, Casey-Ford RG, Jes SP, Thomas GP, Rita C, David CQ, et al. The queen is not a pacemaker in the small-colony wasps polistes instabilis and p dominulus. Anim Behav 2006;71(5):1197e203.

[13] Ali E. Turgut, et al. Kobot: A mobile robot designed specifically for swarm robotics research METU-CENG-TR-2007-05

[14] Michael Rubenstein, Radhika Nagpal. Kilobot: A Robotic Module for Demonstrating Behaviors in a Large Scale (210 units) Collective.

[15] Francesco Mondada1, Michael Bonani1, et al. The e-puck, a Robot Designed for Education in Engineering.

[16] James McLurkin, and Jennifer Smith. Distributed Algorithms for Dispersion in Indoor Environments using a Swarm of Autonomous Mobile Robots.

[17] Elizabeth Jensen and Maria Gini. Rolling Dispersion for Robot Teams 